\title{
A NOTE ON CONSTRUCTIVE METHODS IN BANACH ALGEBRAS
}

PAUL J. COHEN ${ }^{1}$

There are several well-known applications of Banach algebras to questions in classical analysis, the most notable being the Wiener Tauberian theorem. This theorem of course was given a classical proof by Wiener, but the abstract proof in some ways appears simpler and free of computations. The abstract proof has the disadvantage, however, of not yielding a constructive method for obtaining the object whose existence is asserted by the theorem, nor of giving numerical bounds for certain quantities. The origin of this deficiency lies in the use of the axiom of choice. Beurling has given a simple proof of Wiener's theorem on reciprocals of absolutely convergent Fourier series which takes advantage of Banach algebra ideas, but which is purely classical and constructive. It, however, uses special properties of Fourier series which are not used in the abstract proof. The Banach algebra proof is essentially a restatement of the fact that in an algebra with an identity either a set of elements is contained in a maximal ideal or else 1 is a linear combination of them. This principle can be applied to the algebras of absolutely convergent Fourier series, absolutely convergent Taylor series, or functions continuous on the disc, analytic in the interior. We prefer to illustrate our approach by applying it to the third situation. Namely, ${ }^{2}$

THEOREM. If $f_{i}, 1 \leqq i \leqq n$ are continuous functions defined for $|z| \leqq 1$, analytic for $|z|<1$, which are never simultaneously zero, then there exist functions $g_{i}$, analytic in the disc and continuous on the boundary such that $\sum f_{i} g_{i} \equiv 1$.

We shall give a classical proof of this theorem which explicitly yields the functions $g_{i}$. This will be done by means of a fairly simple program which we use to translate abstract proofs into classical proofs.

Let $A$ denote the algebra of functions $f(z)$, analytic for $|z|<1$, continuous for $|z| \leqq 1$, where $\|f\|=\sup |f|$. We review first the standard

\footnotetext{
Received by the editors November 18, 1959, and, in revised form, February 26, 1960.

1 This research has been supported in part by the Office of Naval Research.

2 The referee has pointed out that a classical proof has already been given by $L$. Carleson [1]. It is quite different from the above proof which yields corresponding classical proofs for the other cases mentioned above. I should like to thank J. Wermer for suggesting the possibility of a constructive version of the Banach algebra proof.
} 
abstract proof. We let $I$ denote the ideal generated by $f_{i}$, i.e. the set of all functions of the form $\sum f_{i} g_{i}$. We wish to show that 1 belongs to $I$. We assume that $I \neq A$. First, we extend $I$ to a maximal ideal $J$, invoking the axiom of choice. Second, we observe that $J$ is closed. Third, we form the quotient Banach algebra $A / J$. Fourth, we use the Gelfand-Mazur theorem to deduce that there is a homomorphism of $A$ onto the complexes sending $J$, and hence $f_{i}$, into zero. Finally, we show that any such homomorphism is given by evaluation at some point of the disc, which is a contradiction.

We note that step two above is accomplished by the following simple device, which we can expect to occur in any classical proof.

Lemma. If $\|f\|<1$, then $1-f$ is invertible in $A$.

Proof. $g=1+f+f^{2}+\cdots$ clearly converges in $A$, and $g(1-f)=1$. Step four, the Gelfand-Mazur theorem, is proved by observing that in a normed division algebra $B$, if $z \in B$, and $z \neq \lambda e$ for any complex number $\lambda$, where $e$ is the identity element, then $(z-\lambda e)^{-1}$ is an analytic function of $\lambda$. Since as $\lambda$ tends to infinity this quantity approaches zero, by Liouville's theorem it is identically zero which is absurd, so that $B$ coincides with the multiples of $e$. Liouville's theorem is in turn proved by the Cauchy integral formula taken over a large circle. With these remarks we shall now prove the theorem.

We now denote by $I$ the closure of the ideal generated by $f_{i}$. If 1 belongs to $I$, then 1 is a linear combination of $f_{i}$, since if $1+h=\sum g_{i} f_{i}$, $\|h\|<1$, then $1+h$ is invertible and so $1=\sum(1+h)^{-1} g_{i} f_{i}$. Assume then that $f_{i}$ have no common zero in $|z| \leqq 1$. We first show that for every $\lambda$, there exists $h$ in $A$ such that $(z-\lambda) h \equiv 1$ modulo $I$. If $|\lambda|>1$, then $1 /(z-\lambda)$ belongs to $A$ and so we may take $h$ equal to it. If $|\lambda| \leqq 1$, then we have say $f_{1}(\lambda) \neq 0$. Now the polynomials in $z$ are dense in $A$, a fact which is used in the last step of the abstract proof, and may be proved by taking Fejér means. Thus there is a polynomial $\tilde{f}(z)$, such that $\| \tilde{f}-f_{1}||<\left|f_{1}(\lambda)\right| / 4$. For some $k$ in $A$ we clearly have

$$
(z-\lambda) k(z)=\tilde{f}(z)-\tilde{f}(\lambda),
$$

or

$$
(z-\lambda) k(z)=-\tilde{f}(\lambda)+\left(\tilde{f}(z)-f_{1}(z)\right) \text { modulo } I .
$$

Since $\left\|\tilde{f}-f_{1}\right\|<|\tilde{f}(\lambda)|$, the lemma shows that the right side of (2) is invertible in $A$, so that for some $h$ we have

$$
(z-\lambda) h(z) \equiv 1 \text { modulo } I \text {. }
$$

If (3) holds for a certain $\lambda$, then if we set 


$$
h(s, z)=h(z)(1-(s-\lambda) h(z))^{-1}
$$

for

$$
|s-\lambda|<\|h\|^{-1},
$$

then $h(s, z)$ is well-defined and

$$
(z-s) h(s, z) \equiv 1 \text { modulo I }
$$

since

$$
\begin{aligned}
(z-s) h(s, z) & =[(z-\lambda)-(s-\lambda)] h(z)(1-(s-\lambda) h(z))^{-1} \\
& \equiv(1-(s-\lambda) h(z))(1-(s-\lambda) h(z))^{-1} \\
& \equiv 1 .
\end{aligned}
$$

In the neighborhood defined by (5), for each fixed $z, h(s, z)$ is clearly analytic in $s$. We have now essentially copied the first part of the proof of the Gelfand-Mazur theorem.

Let $\Gamma$ denote the circle $|s|=3$. We form a fine grid so that the interior of $\Gamma$ is the union of regions $C_{j}$, bounded by simple closed curves $\gamma_{j}$, and so that for each $C_{j}$, we have a function $h_{j}(s, z)$ such that (6) holds for $s$ in the neighborhood of $C_{j}$. We orient $\gamma_{j}$ coherently with $\Gamma$. Set

$$
T(z)=\sum_{j} \frac{1}{2 \pi i} \int_{\gamma_{j}} \frac{h_{j}(s, z)}{s} d s .
$$

We assume here that no $\gamma_{j}$ passes through the origin. If $C_{0}$ is the unique $C_{j}$ containing the origin clearly

$$
T(z)=h_{0}(0, z),
$$

and

$$
z T(z) \equiv 1 .
$$

On the other hand along each curve $\boldsymbol{\gamma}_{j}$, we have at least two functions $h_{j_{1}}(s, z)$ and $h_{j_{2}}(s, z)$ defined. But,

$$
(z-s)\left(h_{j_{1}}(s, z)-h_{j_{2}}(s, z)\right) \equiv 0
$$

so multiplying by $h_{j_{1}}(s, z)$ and using (6)

$$
h_{j_{1}}(s, z) \equiv h_{j_{2}}(s, z) \text {. }
$$

Hence we see that

$$
T(z)=\frac{1}{2 \pi i} \int_{\Gamma}(z-s)^{-1} \frac{d s}{s} .
$$


The right side of (12) is zero for $|z| \leqq 1$; hence $T \in I, z T \in I$, but $z T-1 \in I$ by (9), so $1 \in I$. We note that since $I$ is closed, the integration of (11) to prove (12) is justified.

The above proof will also serve as a model to prove Wiener's theorem on the reciprocal of absolutely convergent Fourier series. It can be used to get the following bound:

If $f(z)=\sum_{n=-\infty}^{\infty} c_{n} z^{n}$, and $\sum_{|n|>N}\left|c_{n}\right|<\alpha / 4$, where $\alpha=\min |f(z)|$, then $\|1 / f\|$ can be bounded in terms of $\alpha$ and $\sum_{|n|<N}\left|n c_{n}\right|$.

We now turn to a theorem of Wermer [3], which was given a short abstract proof by Hoffman and Singer [2].

Theorem. Let $f$ be a continuous function on $|z|=1$ with Fourier series $\sum_{n=-\infty}^{\infty} c_{n} z^{n}$. If for some $n<0, c_{n} \neq 0$, then every continuous function on $|z|=1$ can be uniformly approximated by polynomials in $z$ and $f$.

We give a very simple proof which can be regarded as a translation of the proof in [2]. It cannot be said to be simpler, but avoids the axiom of choice and is constructive in the same sense as our previous proof. We may assume that for some $n>0, c_{-n}=1$. The Fourier series for $z^{n} f$ has constant term 1 , and since the polynomials in $z$ and $\bar{z}$ are dense in the space of all continuous functions we have that $z^{n} f$ may be approximated by such a polynomial so that

$$
z^{n} f=1+z g_{1}+\bar{z} \bar{g}_{2}+h
$$

where $g_{1}$ and $g_{2}$ are polynomials in $z$, and $h$ is a continuous function such that $|h|<1 / 2$. Assume

$$
\left|z g_{2}-\bar{z} \bar{g}_{2}\right| \leqq M
$$

for some constant $M$. Since the quantity occurring in (14) is purely imaginary, we have for all $\delta>0$,

$$
\left|1+\delta\left(z g_{2}-\bar{z} \bar{g}_{2}\right)\right| \leqq 1+\delta^{2} M^{2} .
$$

Substituting from (13) we have

$$
\left|1+\delta+z\left(\delta g_{2}+\delta g_{1}-\delta z^{n-1} f\right)+\delta h\right| \leqq 1+\delta^{2} M^{2}
$$

or

$$
|1+\delta+z j| \leqq 1+\delta^{2} M^{2}+\delta / 2
$$

where $j$ is a polynomial in $z$ and $f$. For sufficiently small $\delta$,

$$
1+\delta^{2} M^{2}+\delta / 2<1+\delta
$$

so that by (17) and the lemma, $z j$ is invertible in the algebra $B$ which is the uniform closure of polynomials of $z$ and $f$, so that finally $1 / z$ 
$=\bar{z} \in B$, and since $z \in B, B$ is the algebra of all continuous functions on the circle $|z|=1$.

\section{REFERENCES}

1. L. Carleson, On bounded analytic functions and closure problems, Ark. Mat. vol. 2 (1954) pp. 283-291.

2. K. Hoffman and I. M. Singer, Maximal subalgebras of $C(\Gamma)$, Amer. J. Math. vol. 79 (1957) pp. 295-305.

3. John Wermer, On algebras of continuous functions, Proc. Amer. Math. Soc. vol. 4 (1953) pp. 866-869.

Massachusetts Institute of Technology and

INSTITUTE fOR ADVANCEd STUdY 